See discussions, stats, and author profiles for this publication at: https://www.researchgate.net/publication/252841850

\title{
Global Optimization for Motion Estimation with Applications to Ultrasound Videos of Carotid Artery Plaques
}

Conference Paper in Proceedings of SPIE - The International Society for Optical Engineering · March 2010

DOI: $10.1117 / 12.844496$

CITATION

1

6 authors, including:

Marios S Pattichis

University of New Mexico

334 PUBLICATIONS 3,291 CITATIONS

SEE PROFILE

C. S. Pattichis

University of Cyprus

431 PUBLICATIONS 5,663 CITATIONS

SEE PROFILE

Some of the authors of this publication are also working on these related projects:

AOLME Project View project

Project Prehospital Health Care Management Platform View project
READS

40

Christos P Loizou

University of Cyprus

155 PUBLICATIONS 1,811 CITATIONS

SEE PROFILE 


\title{
Global Optimization for Motion Estimation with Applications to Ultrasound Videos of Carotid Artery Plaques
}

\author{
Sergio Murillo $^{\mathrm{ab}}$, Marios Pattichis ${ }^{\mathrm{a}}$, Peter Soliz ${ }^{\mathrm{b}}$, Simon Barriga ${ }^{\mathrm{b}}$, C.P. Loizou ${ }^{\mathrm{c}}$, and C.S. Pattichis ${ }^{\mathrm{d}}$ \\ ${ }^{a}$ Department of Electrical and Computer Engineering-University of New Mexico, Albuquerque, \\ NM-87131 \\ ${ }^{\mathrm{b}}$ VisionQuest Biomedical, Albuquerque, NM-87106 \\ ${ }^{\mathrm{c}}$ Department of Computer Science, Intercollege, Limassol Campus, CY-3507 Limassol, Cyprus. \\ ${ }^{\mathrm{d}}$ Department of Computer Science, University of Cyprus, CY1678 Nicosia, Cyprus.
}

\begin{abstract}
Motion estimation from digital video is an ill-posed problem that requires a regularization approach. Regularization introduces a smoothness constraint that can reduce the resolution of the velocity estimates. The problem is further complicated for ultrasound videos (US), where speckle noise levels can be significant. Motion estimation using optical flow models requires the modification of several parameters to satisfy the optical flow constraint as well as the level of imposed smoothness. Furthermore, except in simulations or mostly unrealistic cases, there is no ground truth to use for validating the velocity estimates. This problem is present in all real video sequences that are used as input to motion estimation algorithms. It is also an open problem in biomedical applications like motion analysis of US of carotid artery (CA) plaques. In this paper, we study the problem of obtaining reliable ultrasound video motion estimates for atherosclerotic plaques for use in clinical diagnosis.

A global optimization framework for motion parameter optimization is presented. This framework uses actual carotid artery motions to provide optimal parameter values for a variety of motions and is tested on ten different US videos using two different motion estimation techniques.
\end{abstract}

Keywords: Motion estimation, ultrasound videos, carotid artery plaques, global optimization.

\section{INTRODUCTION}

Ultrasound videos of the carotid artery provide significant clinical information for the characterization of atherosclerotic plaques. We focus on the estimation of plaque motion. Block matching using cross correlation is the most common technique [1-4]. However, block matching does not provide motion estimation resolution at the pixel level. Since it provides a single motion vector for an entire block of pixels; it is an efficient approach for use in video coding. On the other hand, it is not accurate enough for video image analysis applications.

To provide high-resolution motion estimates, it is standard practice to use an optical flow method. Motion estimation using optical flow models has been proven to be a useful non-invasive tool for diagnosis of symptomatic and asymptomatic atherosclerotic plaques [5]. Results using this increased resolution will yield better understanding of the mechanics and deformations of atherosclerotic plaques during the cardiac cycle, and can be potentially used to predict their rupture.

Optical flow models assume the conservation of the image brightness along the motion path. This is known as the optical flow constraint (OFC), and leads to a single equation per pixel for estimating two velocity components. Furthermore, the OFC only constraints the motion in the direction of the local image gradient [6]. In several optical flow methods, a smoothness constraint is added in order to produce a second equation [7-10]. The relative weight between the two constraints in the optical flow model is controlled by a regularization parameter, and additional motion estimation parameters can also be used. For differential techniques [6], we also need to determine how much smoothing must be applied to the video sequence before computing the image intensity gradient (in addition to the regularization parameter). Thus, picking correct values for the model parameters is a critical task. 
Previous work [11] for estimating the regularization parameter value is based on formulating noise models and minimizing a confidence measure. Another approach $[12,13]$ relies on assuming prior information about the velocity field probability distribution and uses a Bayesian framework to solve for the regularization parameter using a maximum a posteriori estimator (MAP). These approaches work with idealized simulations that do not capture the complexity of spatio-temporal deformations of clinical motion.

When available, the preferred approach is to use ground truth methods to asses the accuracy and also to set the values of the model parameters [6, 14-17]. Unfortunately, this approach is limited to simulations or simple physical motions. When a technique is applied to a video sequence that lacks ground truth, performance can only be assessed from subjective observations. This problem becomes more relevant in biomedical applications like plaque motion analysis where accurate pixel velocities are needed to gain more information about atherosclerotic plaques that may become symptomatic. Our approach is to develop a realistic motion simulator as a first step to tune up the motion estimation parameters to the underlying video motion. This approach is an extension of our prior work presented in [18-20] where the motion simulator was developed to resemble observed carotid artery displacements and to satisfy clinical expectations. The simulator works by imposing a periodic motion of different amplitudes and frequencies on plaque and artery wall. The periodicity is a consequence of the cardiac cycle and it has been observed in our previous work [18-20] as well as in the work of Stoitsis [2, 3, 21] and Golemati [1]. After simulations, a global optimization algorithm that uses a branch and bound strategy is applied to the synthetic video sequences in order to find the right amount of presmoothing prior to image gradient calculation and also to find the optimal value of the regularization parameter. Once the optimal parameters are calculated, they are applied to the clinical videos to estimate the velocity field. After motion estimation, a Kalman filter is used to reconstruct pixel trajectories, and followed by principal component analysis (PCA) applied to the trajectories in order to separate motion patterns.

The paper is organized in the following manner: The methodology is presented in section 2 . The results, section 3 , are followed by a discussion in section 4, and conclusions and future work are provided in section 5 .

\section{METHODOLOGY}

The proposed methodology relies on fine tuning the motion estimation algorithms to the underlying video motion using a realistic motion simulator along with a global optimization algorithm. Thus, optimization is used to estimate the optimal motion parameters that will produce accurate pixel velocities. After optimization, these parameters are applied to clinical ultrasound sequences and the results of two different motion estimation algorithms are compared and graded. A Kalman filter is used to reconstruct pixel-trajectories and PCA is applied in order to find motion patterns during plaque motion. This methodology is tested on ten ultrasound videos of carotid artery plaques. In what follows, we provide details on the approach.

\subsection{Motion Estimation Techniques}

For motion estimation, the techniques of Horn and Schunk [7] and Lucas and Kanade [22] are considered. These two techniques fall into the category of differential methods because they both need the image intensity gradient to estimate pixel velocities. The main difference between them is how they solve the optical flow model. Horn's technique enforces the smoothness constraint over the entire video image while Lucas uses weighted least squares fit over a small neighborhood to calculate the velocity field. Thus, the former is considered a global solution and the latter a local solution.

In [7], Horn formulated that the pixel intensity brightness values should remain constant along the motion path

$$
I(x, y, t)=I(x+\delta x, y+\delta y, t+\delta t)
$$

Following a Taylor series expansion and neglecting high order terms, equation becomes (1) the OFC

$$
I_{x} u+I_{y} v+I_{t}=0
$$


where $\vec{v}=(u, v)$ denote velocity estimates at pixel $(x, y)$. Global deviations from the OFC are measured by the following energy functional:

$$
E_{O F C}=\iint_{\Omega}\left(I_{x} u+I_{y} v+I_{t}\right)^{2} d \Omega
$$

which is minimized over the entire image domain $\Omega$. Here, (3) is ill-posed since it provides a single equation in two unknowns (per pixel). More specifically, in what is known as the aperture problem, only the motion in the direction of the local intensity gradient can be calculated using (2). Thus, a regularization assumption that imposes a smoothness constraint in the velocity field is used to fully estimate video motion. The idea behind the smoothness constraint is that nearby pixels have similar velocities. This is formulated in terms of the derivatives of the velocity field:

$$
E_{S}=\iint_{\Omega}\left(u_{x}^{2}+u_{y}^{2}+v_{x}^{2}+v_{y}^{2}\right) d \Omega
$$

The solution to the constant brightness model (CBM) is the velocity field that minimizes the sum of errors in the following model:

$$
\begin{aligned}
E_{C B M} & =E_{O F C}+\alpha^{2} E_{S} \\
E_{O F C} & =\iint_{\Omega}\left(I_{x} u+I_{y} v+I_{t}\right)^{2} d \Omega \\
E_{S} & =\iint_{\Omega}\left(u_{x}^{2}+u_{y}^{2}+v_{x}^{2}+v_{y}^{2}\right) d \Omega
\end{aligned}
$$

Instead of imposed global constraints to estimate pixel velocities, Lucas and Kanade [22] proposed solving (2) in local neighborhoods assuming a constant velocity model and applying least squares (LS). The LS was applied to a weighted version of the OFC in a way that more influence was given to measurements at the center of the neighborhood than those at the periphery:

$$
\sum_{(x, y) \in \Gamma}\left[W(x, y)\left(I_{x} u+I_{y} v+I_{t}\right)^{2}\right]
$$

The weight function, $\mathrm{W}(\mathrm{x}, \mathrm{y})$, is separable and its support determines the size of the neighborhood $\Gamma$.

\subsection{Carotid artery plaque motion simulator}

Periodic motion of the artery can be observed in ultrasound videos [1-3, 18, 19, 21] and comes from the fact that the artery follows the cardiac cycle. Thus a periodic motion along with its discontinuities is imposed to the video frames using a Fourier series expansion to create a set of coordinate transformations that generate synthetic motion. The amplitude and frequencies are changed in four simulations so that the motion estimation techniques can be tuned up to different motion patterns that reflect the motion present in the ultrasound videos.

The coordinate transformation equation that displaces the image intensities in the axial and radial directions at every frame is given by:

$$
\begin{aligned}
& a(x, y, t)=A_{a} \sin \left(\frac{2 \pi}{N} f_{h} t\right)+\frac{A_{a}}{2} \sin \left(\frac{2 \pi}{N}\left(2 f_{h}\right) t\right)+\frac{A_{a}}{3} \sin \left(\frac{2 \pi}{N}\left(3 f_{h}\right) t\right), \text { and } \\
& r(x, y, t)=A_{r} \sin \left(\frac{2 \pi}{N} f_{r} t\right)+\frac{A_{r}}{2} \sin \left(\frac{2 \pi}{N}\left(2 f_{r}\right) t\right)+\frac{A_{r}}{3} \sin \left(\frac{2 \pi}{N}\left(3 f_{r}\right) t\right)
\end{aligned}
$$

In (7), the amplitude of the axial motion component is given by $A_{a}$ while the radial amplitude is represented by $A_{r}$. Similarly, radial and axial frequencies are referred by $f_{r}$, and $f_{a} . N$ is the total number of frames in the simulation and 
$t$ is the index time parameter. Note that amplitudes are decaying at a rate that is inversely proportional to the fundamental frequency simulating motion discontinuities that can be induced by random events like for example lung motions [18-20]. The simulator works under the assumption that plaques with low degree of stenosis move at larger amplitudes than plaques with high stenosis since the narrowing of the artery increases with the stenosis degree. The simulation parameters are given in table 1 , and figure 1 shows an ultrasound video frame with the axial and radial directions.

Table 1. Synthetic motion simulation parameters. Amplitudes, frequencies, and degree of stenosis change for each simulation case. These parameters are set to cover different motion patterns observed in clinical ultrasound videos.

Synthetic Motion Parameters

\begin{tabular}{lllll}
\hline \hline Video Number & $\# 1$ & $\# 2$ & $\# 3$ & $\# 4$ \\
Stenosis (\%) & $52 \%$ & $26 \%$ & $73 \%$ & $60 \%$ \\
Axial Frequency (cycles per video length) & 5 & 5 & 5 & 3 \\
Radial Frequency (cycles per video length) & 4 & 4.5 & 6 & 2.5 \\
Axial Motion Amplitude (mm) & 0.68 & 0.83 & 2.25 & 0.1 \\
Radial Amplitude (mm) & 0.45 & 0.45 & 0.3 & 0.08 \\
Number of frames & 300 & 300 & 300 & 200 \\
\hline \hline
\end{tabular}

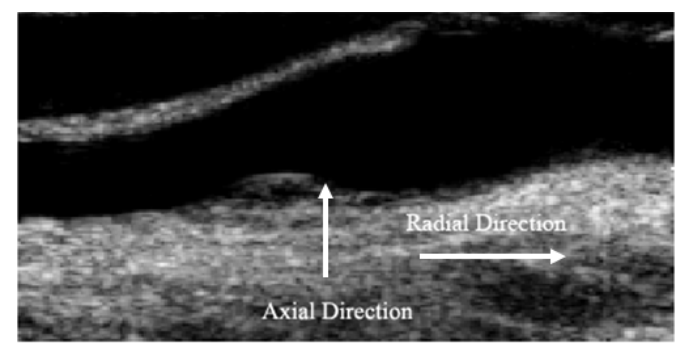

Figure 1. A single frame of a carotid ultrasound image. The figure illustrates the axial and radial directions

\subsection{Speckle Simulation}

The proposed methodology considers that speckle is a major source of inaccuracies when estimating ultrasound video motion due to loss of coherence that causes changes in image intensities. Thus, proper optimization is carried out to find the limits of the motion estimation techniques by corrupting the simulations with different levels of speckle signal to noise rations (SNR). This is used to calculate the set of optimal input parameters that produce the most accurate velocity estimates at a given SNR. The simulated examples were corrupted with speckle noise following the model proposed in [23]. This model assumes that after logarithmic compression of the envelop detected signal, speckle can be modeled as independent and identically distributed (i.i.d) additive white Gaussian noise (AWG).

\subsection{Global Optimization}

The techniques presented in section 2.1 are known as differential methods because they need the intensity gradient to calculate pixel velocities. Quantization error and noise affect the calculation of the image intensity derivatives. Thus, pre-smoothing of the video frames is necessary prior differentiation. The amount of smoothing is controlled by the spread of a Gaussian filter. Too much smoothing will destroy local image structure and not enough smoothing will produce noisy intensity gradients. Another factor that affects the accuracy of the velocity estimates is the relative weight of the smoothness constraint, $E_{S}$, relative to the OFC. This relative weight is controlled by the $\alpha$ parameter in equation (5). If image intensity measurements are accurate, this parameter should be small and large if they are noisy [24]. Moreover, $E_{S}$ only vanishes for constant velocities, i.e. $u_{x}=u_{y}=v_{x}=v_{y}=0$. Clearly, this is never satisfied for clinical applications of interest and the smoothness constraint is non zero. Optimizing the weight of the smoothness constraint is 
thus a difficult and a non-trivial task. For Horn and Schunk [24], optimization will be carried out for the spread of the Gaussian pre-smoothing filter, $\sigma$, and the regularization parameter (coefficient of the smoothness constraint), $E_{S}$, in model (5). For Lucas [22], optimization searches for the correct amount of smoothing of the input sequence controlled by the spread of the Gaussian pre-smoothing filter, $\sigma$.

We use branch and bound for global optimization. Here, branch and bound is used to partition the feasible set into disjoint set intervals. Over each set interval, lower and upper bounds of the minimum objective function value are determined (bounding). Set intervals of the feasible set with lower bounds larger than the best upper bound found at a certain stage are deleted from further consideration (pruning) because these set intervals of the domain cannot contain the optimum. The objective function which will be minimized using this method is the mean square error (MSE) of the velocity magnitudes given by:

$$
\begin{aligned}
\text { GTMag } & =\sqrt{u_{g t}^{2}+v_{g t}^{2}} \\
\text { ESTMag } & =\sqrt{u_{\text {est }}^{2}(\vec{p})+v_{\text {est }}^{2}(\vec{p})} \\
f(\vec{p}) & =\frac{1}{M N} \sum_{i=0}^{M-1} \sum_{j=0}^{N-1}\left(\text { ESTMag }_{i j}(\vec{p})-\text { GTMag }_{i j}\right)^{2}
\end{aligned}
$$

The vector $\vec{p}$ is composed of the motion estimation parameters that govern the optical flow estimates and varies according to each method. Now, consider the following minimization problem:

$$
\min _{\text {s.t. } a \leq x \leq b} f(\vec{p}) \text {, }
$$

where $a=\left(a_{1}, \ldots, a_{n}\right)^{T}, b=\left(b_{1}, \ldots, b_{n}\right)^{T} \in R^{n}, a<b$, and $f: R=\{x: a \leq x \leq b\} \rightarrow R$ is assumed to be Lipschitzian on $D$ with Lipschitz constant $L$. Then, the problem is called a Lipschitz optimization problem [25].

A real value function $f$ is called Lipschitzian, or a Lipschitz function, on a set $P \subseteq R^{n}$ if there is a (Lipschitz) constant $L=L(f, P)>0$ such that $\left|f\left(x_{2}\right)-f\left(x_{1}\right)\right| \leq L\left\|x_{2}-x_{1}\right\|$ for all $x_{1}, x_{2} \in P$. If $f$ is Lipschitz on $P$ with constant $L$, then $f$ is also Lipschitzian on $P$ with all constants $L^{\prime}>L$. When $P$ is a real interval, $L$ provides an upper bound for the absolute value of the slope of any line joining two points on the graph of $f$. Now, let $P \subset R^{n}$ be convex, and let $f$ be continuously differentiable on an open set containing $P$ with bounded gradient on $P$. Then, $f$ is Lipschitzian on $P$ with constant: $L=\sup \{\|\nabla f(x)\|: x \in P\}$. The supremum can be replaced by the maximum of $\|\nabla f(x)\|$ over $P$, when $P$ is a bounded closet set and $\nabla f(x)$ is continuous and bounded. An iterative algorithm based on the described formulation is applied to find the region of the parameter space that minimizes MSE of the synthetic simulations.

\subsection{Kalman Filtering}

Once the optimal parameters are calculated, they are used in clinical ultrasound videos in order to estimate the corresponding velocity field. We use a Kalman filter to track plaque motion. The goal is to predict the position of a pixel given its initial coordinates and velocity estimates throughout the video sequence. The linear models for trajectory reconstruction applied independently to the axial and radial directions are given by:

$$
\begin{array}{cc}
{\left[\begin{array}{l}
x(k+1) \\
u(k+1)
\end{array}\right]=\left[\begin{array}{ll}
1 & 1 \\
0 & 1
\end{array}\right]\left[\begin{array}{l}
x(k) \\
u(k)
\end{array}\right]+\xi_{h}(k)} & \text { and } \quad\left[\begin{array}{l}
y(k+1) \\
v(k+1)
\end{array}\right]=\left[\begin{array}{ll}
1 & 1 \\
0 & 1
\end{array}\right]\left[\begin{array}{l}
y(k) \\
v(k)
\end{array}\right]+\xi_{v}(k) \\
{[u(k)]=\left[\begin{array}{ll}
0 & 1
\end{array}\right]\left[\begin{array}{c}
x(k) \\
u_{t}(k)
\end{array}\right]+\omega_{h}(k),} & {[v(k)]=\left[\begin{array}{ll}
0 & 1
\end{array}\right]\left[\begin{array}{c}
y(k) \\
v_{t}(k)
\end{array}\right]+\omega_{v}(k) .}
\end{array}
$$


The state vector is composed of position and velocity, and the index $k$ indicates discrete time intervals. The velocity estimates, $u(k)$ and $v(k)$, are the observations of the Kalman filter. The vector $\left[\begin{array}{ll}u_{t}(k) & v_{t}(k)\end{array}\right]^{T}$ represents the motion vector. Process noise and observation noise are modeled as zero-mean, uncorrelated, white-noise $\xi(k)$, and $\omega(k)$ processes respectively. The covariance matrix of the process noise and variance of the observation noise are given by:

\subsection{Principal Component Analysis}

$$
\begin{aligned}
E\left[\xi(k) \xi^{T}(k)\right] & =Q(n)=\sigma_{\xi}^{2}\left[\begin{array}{ll}
1 & 1 \\
1 & 1
\end{array}\right] \text { with } \sigma_{\xi}^{2}=0.5 \text { pixels }^{2} \text {, and } \\
\sigma_{\omega}^{2} & =0.1 \text { (pixels / frame })^{2} .
\end{aligned}
$$

Spatial PCA is applied to the plaque trajectories in order to find patterns from which plaque motion can be characterized. The goal is to differentiate among plaque regions that undergo different motions and identify these patterns along the cardiac cycle. Only the principal components that account for $90 \%$ of the trajectory variance are selected.

\section{RESULTS}

\subsection{Synthetic video simulation results}

Synthetic simulations results are presented in the form of contour plots and tables of the relative error. Note that the parameter space of Horn's technique is two dimensional while that of Lucas's is one dimensional. Thus, Horn's results are presented as 2-D contour plots. The spread of the Gaussian pre-smoothing filter was allowed to vary among $\sigma=0.5-4$ pixels for both techniques. The values of the regularization parameter $\alpha$ varied among $\alpha=1-50$ for Horn's technique.

For each simulation case and motion estimation technique, the goal of the global optimization algorithm is to find a set of parameters that produces the smallest MSE. Intersecting the results of all the simulated cases leads to a common point that will be used as the starting value of the parameters to be used on clinical videos.

Results of Horn's technique are presented figure 2, and tables 2-3. The contour plots indicate the MSE value for a combination of input parameters, and the tables show the maximum relative error of the estimated velocity. For example, table 2 has an error of 0.1 for $\alpha=1.0$ and $\sigma=1.0$. This means that on average, the error is at most $10 \%$ of the maximum ground truth velocity. Figure 3 shows the contour plots for the simulations that include speckle. Horn's technique was used in this case with the error measured in $\mathrm{mm} / \mathrm{sec}$. Figure 4 shows the relative error as a function of the Gaussian pre-smoothing step for Lucas technique.

The information of the contour plots and relative error tables is later used to select a set of parameter values that produces the smallest error among all the simulated cases. These values are used as a starting point when estimated velocities on clinical videos.

Table 2. Relative velocity estimation error for simulation cardiac4. Relative errors up to $10 \%$ are considered acceptable.

\begin{tabular}{ccccc} 
Relative Velocity Error of Simulated Video \# 4. \\
Max Vgt $=0.06 \mathrm{~mm} / \mathrm{sec}$ \\
\hline \hline Spread Smoothing Filter \\
Reg. Par. & 0.75 & 1.0 & 1.25 & 1.5 \\
\hline \hline 1.0 & 0.10 & 0.10 & 0.21 & 0.13 \\
1.4 & 0.12 & 0.13 & 0.15 & 0.18 \\
1.9 & 0.14 & 0.16 & 0.20 & 0.22 \\
2.5 & 0.18 & 0.21 & 0.25 & 0.27 \\
\hline \hline
\end{tabular}




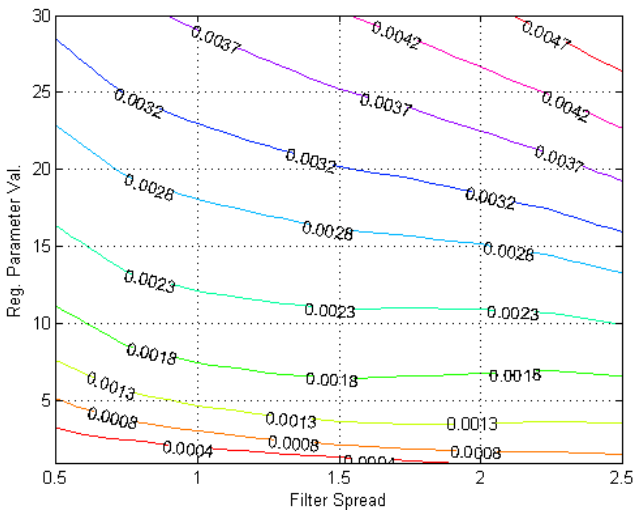

(a)

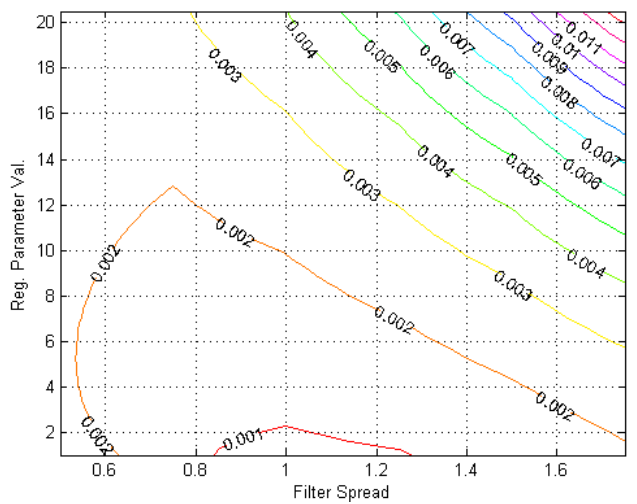

(b)

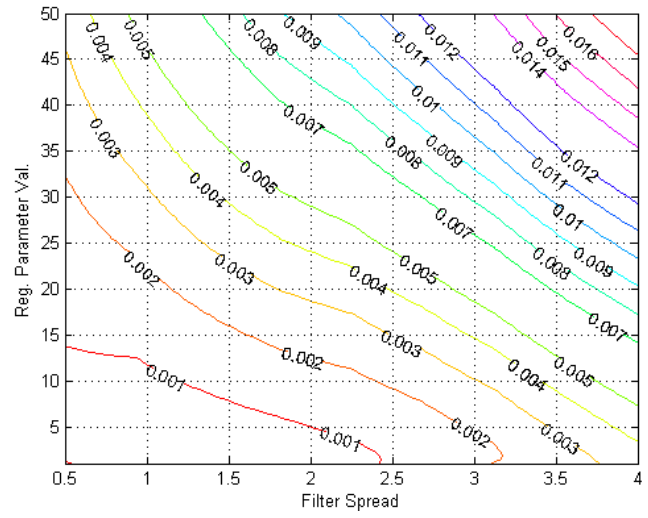

(b)

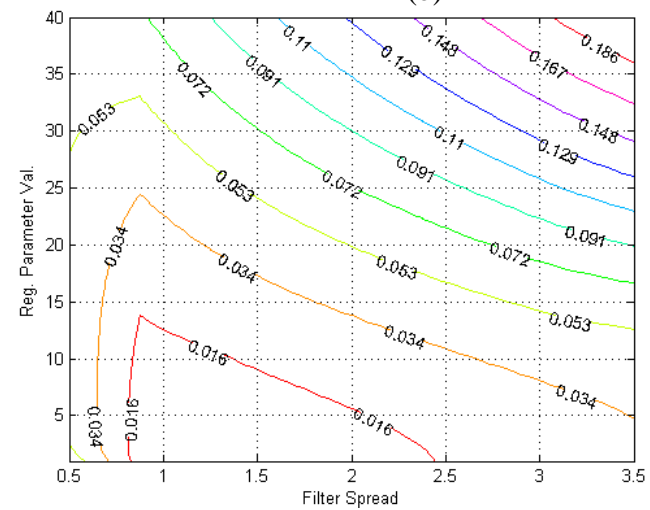

(d)

Figure 2 Contour plots of the velocity MSE for Horn's technique. (a) MSE of simulation cardiac4. (b) MSE of simulation p843. (c) MSE of simulation p815. (d) MSE of simulation p816. Notice how the error increases as the simulated motion increase in amplitude (see table 1). The error is calculated in $\mathrm{mm} / \mathrm{sec}$

Table 3. Relative velocity estimation error for simulation p815. Relative errors up to $10 \%$ are considered acceptable.

Relative Velocity Error of Simulated Video \#1.

Cardiac 4. Max Vgt $=0.28 \mathrm{~mm} / \mathrm{sec}$

\begin{tabular}{cccccc}
\hline \hline & \multicolumn{5}{c}{ Spread Smoothing Filter } \\
Reg. Par. & 0.75 & 1.0 & 1.5 & 1.75 & 2.0 \\
\hline \hline 1.0 & 0.08 & 0.07 & 0.09 & 0.10 & 0.12 \\
2.5 & 0.09 & 0.08 & 0.09 & 0.11 & 0.13 \\
8.6 & 0.09 & 0.10 & 0.13 & 0.15 & 0.17 \\
11.6 & 0.10 & 0.11 & 0.13 & 0.17 & 0.20 \\
\hline \hline
\end{tabular}




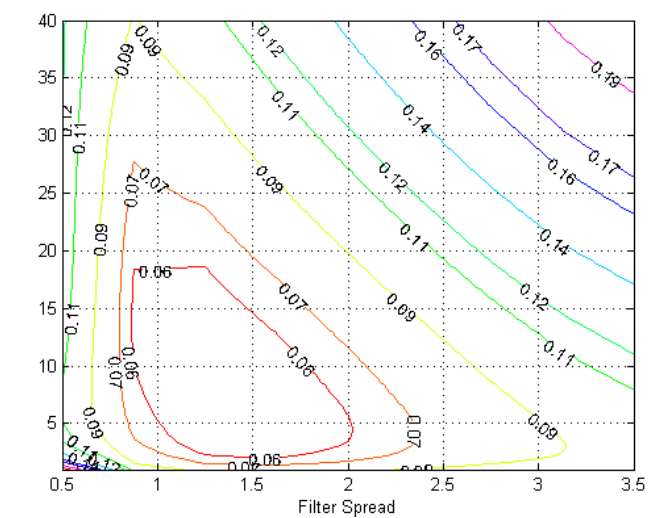

(a)

Figure 3. Contour plots of the MSE for speckle simulations of SNR =10db (a) and $30 \mathrm{db}$ (b). Higher regularization parameter values and filter spreads are necessary to obtain reliable velocity estimates.

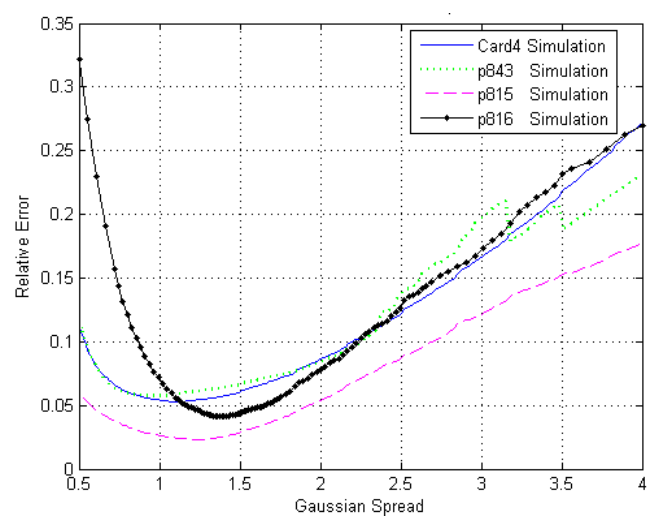

Figure 4. Lucas relative error as a fucntion of the Gaussian pre-smoothing filter. Errors smaller than $10 \%$ are acceptable. Notice how the spread of the filter changes for each simulation (see table 1).

\subsection{Clinical Ultrasound Video Results}

Velocity estimation of clinical ultrasound videos was performed on a set of seven videos. The evaluation of the estimated velocities is done visually by feeding the flow field to a Kalman filter that reconstructs pixel trajectories. The clinical video is then played back with the trajectories over the plaque. This process identifies if the current motion parameters are doing a good job at estimation the true displacement field. When the trajectories lose track of the plaque, a new set of input parameters are used. Speckle is the cause that the parameters cannot track certain plaque regions, thus for these cases the motion parameters are changed to the ones found in the speckle simulation.

Trajectories for a clinical video not used in simulations are shown in figure 5. These trajectories show the motion of points located over the plaque and near the plaque-artery wall boundary. On these examples evidence of periodic motion can be observed. PCA is also performed on the trajectories to identify motion patterns over different plaque regions which can help to better understanding of how the plaque moves during the cardiac cycle and especially at systole and diastole.

\section{DISCUSSION}

\subsection{Global optimization for simulated videos}

Simulation of videos numbered \#4 and \#3 assumed high stenosis values (see table 1). Thus a smooth velocity field of small amplitude was imposed. Six cardiac cycles over three hundred frames with an axial motion amplitude of $2.15 \mathrm{~mm}$ 
, and a radial amplitude of $0.44 \mathrm{~mm}$ were simulated in video \#3. Those parameters translate to a maximum velocity magnitude of approximately $v_{G T \text { (max) }}=0.5 \mathrm{pix} /$ frame or $0.075 \mathrm{~mm} / \mathrm{sec}$. Similarly, two and a half cardiac cycles over two hundred frames with an axial amplitude of $0.15 \mathrm{~mm}$ and a radial amplitude of $0.12 \mathrm{~mm}$ were simulated for video \#4 giving a maximum velocity of approximately $v_{G T(\max )}=0.3 \mathrm{pix} /$ frame or $0.045 \mathrm{~mm} / \mathrm{sec}$. These velocity values are well inside the estimation limits of the optical flow models. Thus, high accuracy is expected from these techniques. For video $\# 1$, with a stenosis value of $52 \%$, the simulated motion was allowed to have higher amplitudes and velocities. The maximum velocity of this video was $v_{G T(\max )}=1.1 \mathrm{pix} /$ frame or $0.165 \mathrm{~mm} / \mathrm{sec}$. Finally, the largest motion and nonsmooth velocity field was simulated for the smallest degree of stenosis. The maximum velocity of video \#2 was $v_{G T(\max )}=1.75 \mathrm{pix} /$ frame or $0.262 \mathrm{~mm} / \mathrm{sec}$. This represented a difficult case since optical flow models depend on finite differences to approximate image gradients along with the small displacement assumption to estimate motion.

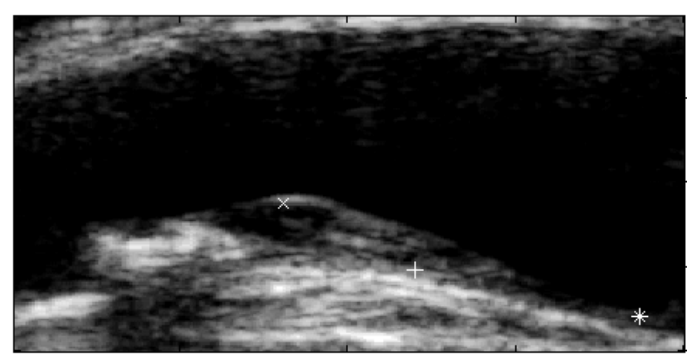

(a)

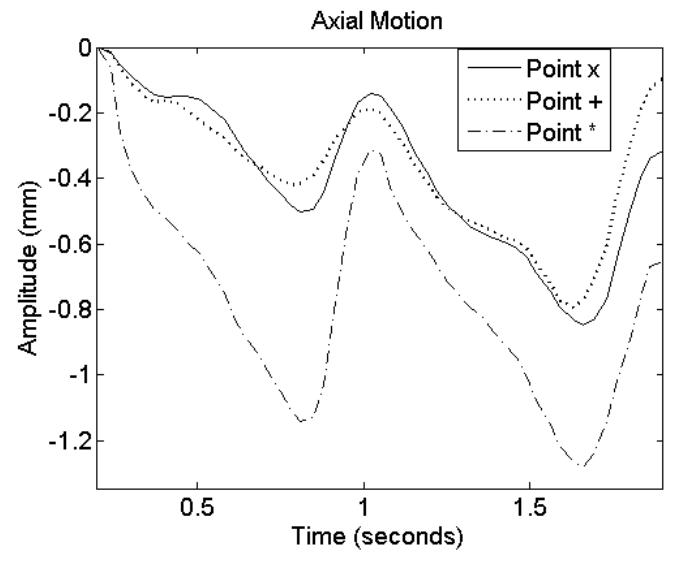

(b)

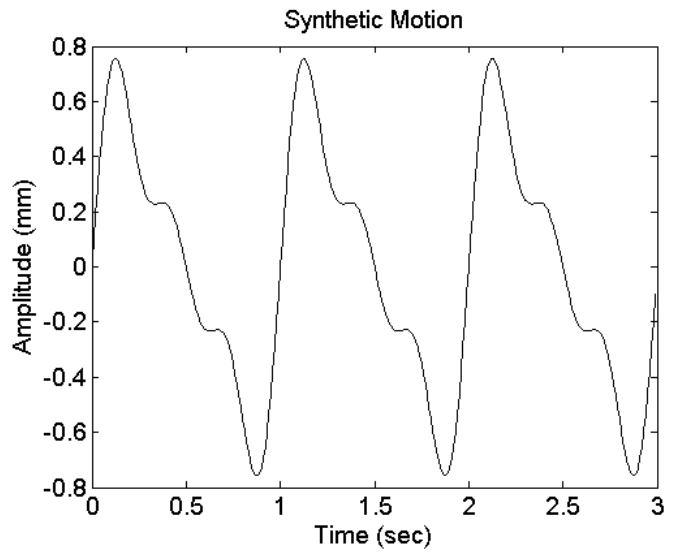

(c)

Figure 5. Estimated trajectories of a clinical ultrasound video. (a) Frame of video p1104 with three selected points. (b) Reconstructed trajectories for the three points shown in fig.5a. (c) Synthetic Motion produced with the simulator.Evidence periodic motion is found in the reconstructed trajectories that approximately resemble the synthetic motion.

Fig. 2 shows the contour plots of the MSE of the velocity magnitude. In all the graphs the error is reduced to ranges of ten to twenty percent of the maximum velocity. Notice how smooth velocity fields (fig. 1a-b) need less smoothing $\sigma$ and smaller values of the regularization parameter $\alpha$ to achieve an optimal region of operation that estimates velocity values within ten percent, i.e. less than $0.007 \mathrm{pix} / \mathrm{sec}$ or $0.004 \mathrm{pix} / \mathrm{sec}$ in the simulated cases, of the maximum velocity. Thus, accurate estimation is achieved when both constraints in (5) have approximately the same relative weight in the model. On the other hand, when the velocity magnitude increases, the estimation error becomes larger (fig.1 c-d). Another interesting result is that if higher spatial smoothing is necessary, the regularization parameter value needs to decrease in order to keep the error from ten to twenty percent of the maximum velocity. On the other hand, higher regularization parameters go with smaller spatial smoothing values. 
Figure 3 shows the contour plots of the MSE of the velocity magnitude estimation at SNR of 10db in fig 3(a) and 30db in 3(b). Optimization was carried over all video frames and as expected, the error is higher for videos of low SNR. The spread of the Gaussian pre-smoothing filter controls the degree of speckle reduction. Videos with smaller SNR require filters with bigger spreads than videos with smaller SNR to produce more accurate pixel velocities. The smallest error is obtained for higher regularization parameter values for videos of low SNR, while for less noisy videos most accurate estimates are obtained for smaller regularization values. This comes from the fact that noisy frames affect the calculation of the image gradient, requiring higher regularization parameters to compensate for these inaccuracies

The parameter set that produces the smallest error for all the simulations is $(\alpha=1.0, \sigma=1.25)$. In the case of Lucas' method the spread of the smoothing filter can vary among $\sigma=0.75-2.25$.

\subsection{Motion estimation of clinical videos}

When estimating velocities of clinical videos it is important to obtain high density fields because it means that the algorithms are working correctly and are able to estimate the velocities for most of the duration of the video. Moreover, the more dense the estimated field, the more information can be assessed from the video. Thus, global techniques are preferred to local methods because they give higher densities.

PCA of clinical video trajectories reveal that in this case there are two motion patterns present during the cardiac cycle. The patterns are discovered by correlating the principal components with all the video trajectories. Then, points with correlation coefficients higher than 0.7 are clustered in a group. The right part of the plaque mainly moves up and down while the left part has a combined horizontal and vertical motion. Fig. 6 shows these plaque regions.

Consistency in the reconstructed trajectories for clinical video \#2 can be observed in fig. 7. This is evidence that when two different motion estimation algorithms produce almost the same estimates, they are correctly estimating the true video motion. When the incorrect input parameters are used on two different methods, it is easy for the methods to produce different and wrong answers, but when the correct parameters are used both methods will most likely converge close to the true velocity field.

The motion parameters obtained with the speckle simulations are able to track the motion for six out of seven videos. For one video, suffering from high levels of speckle decorrelation, we found that it was not possible to successfully estimate the true motion field.

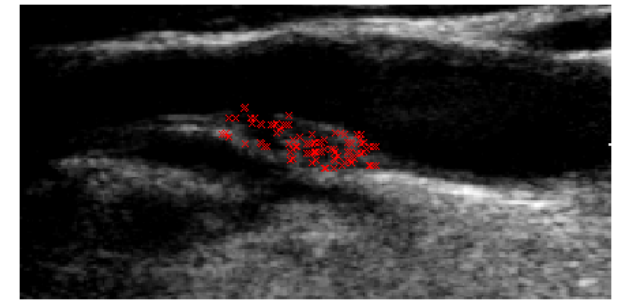

(a)

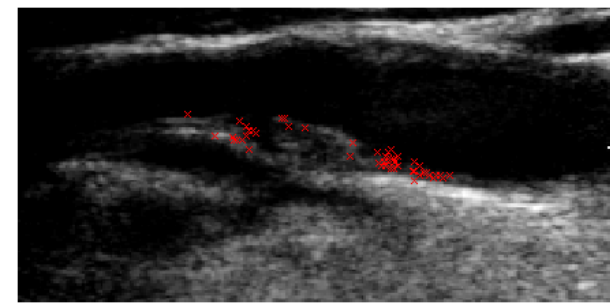

(b)

Figure 6. Regions of a clinical video that exhibit different motion patterns. Identification of these patterns is done with PCA. Points in figure 5a exhibit a combined horizontal and vertical motion, while points in fig. 5b have dominant vertical motion.

\section{CONCLUSIONS AND FUTURE WORK}

The accuracy of the motion estimation is significantly affected by the selection of the model parameters. When working with clinical videos, i.e. without real ground truth, it is very important to select the correct parameter value in order to achieve accurate estimation. Global optimization finds a range of parameter values that can be used on a variety of clinical videos producing accurate velocity estimates. In particular, we have found that a single set of optimal values can produce accurate results for a variety of motions and relatively low noise levels. When noisy levels increase, another set 
of parameters is able to correctly estimate video motion. The velocities estimates can be used to estimate pixel trajectories and analyze the motion of the artery wall and plaque regions which provides important information on how the plaque moves during the cardiac cycle.

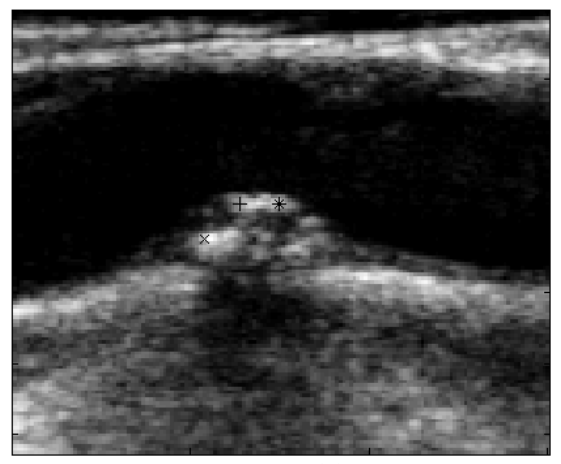

(a)

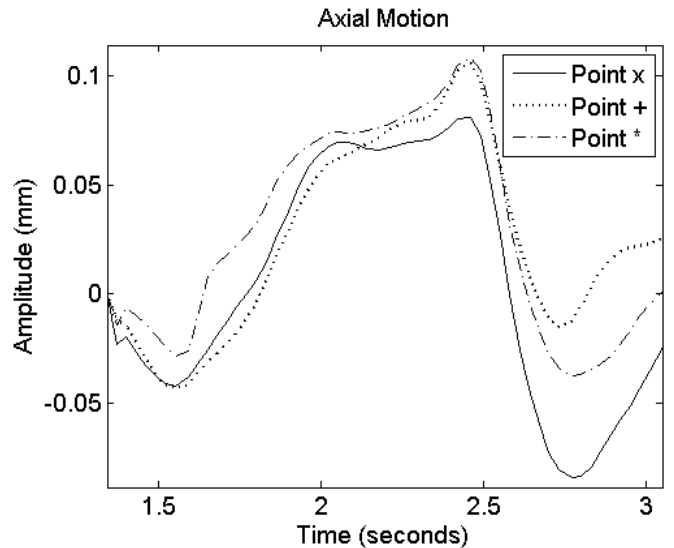

(b)

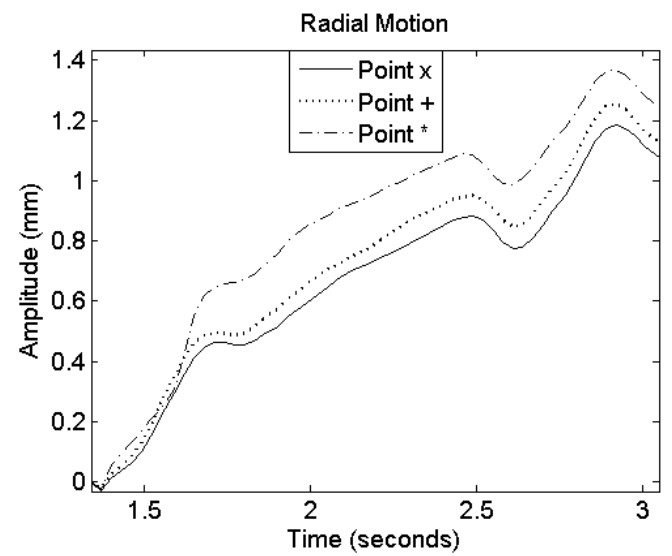

(d)

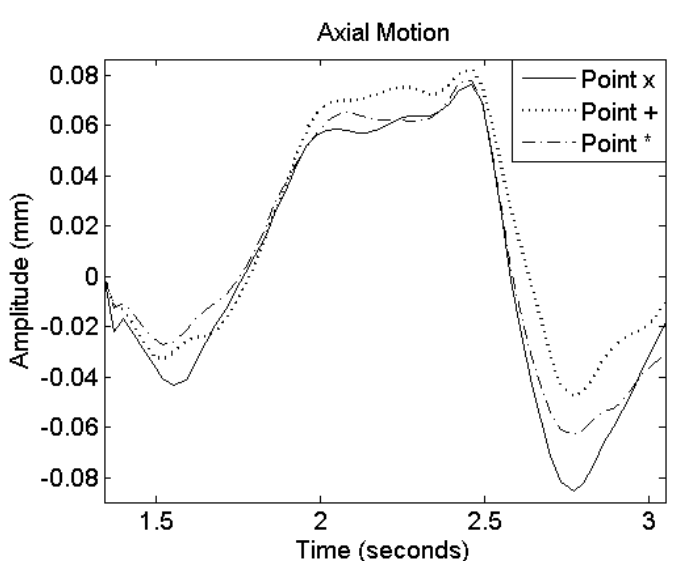

(c)

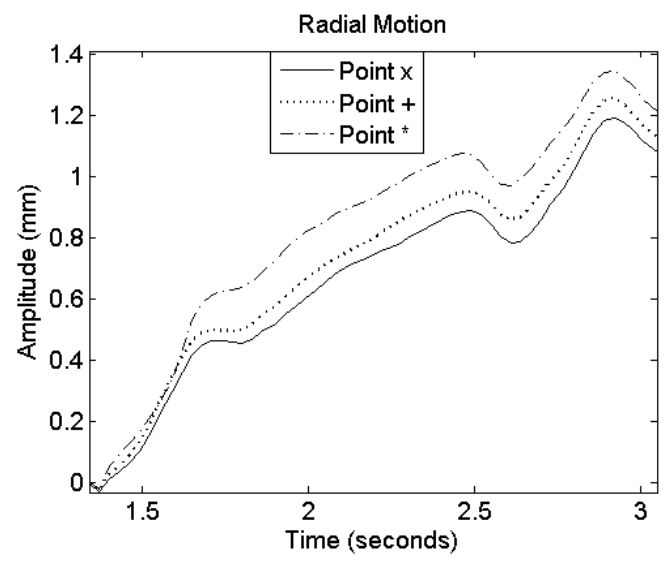

(e)

Figure 7. Reconstructed trajectories of three plaque points using Horn's and Lucas's techniques. (a) Plaque points. (b) Horn axial motion. (c) Lucas axial motion. (d) Horn radial motion. (e) Lucas radial motion. Similar trajectories produced with two different methods indicate that the correct pixel velocities are being calculated.

Future work will be focused on developing reliable confident measures for evaluating pixel velocities when no ground truth is available and exploring the fact that when different methods agree on the same estimation, they are more likely to be providing accurate estimates of the correct velocity field. 


\section{REFERENCES}

[1] Golemati, S., et al., "Comparison of B-mode, M-mode, and Hough Transfrom Methods for Measurement of Arterial Diastolic and Systolic Diameters," in IEEE Eng. in Medicine and Biology 27th Annual Conference. 2005: Shanghai, China.

[2] Stoitsis, J., et al., "A mathematical model of the mechanical deformation of the carotid artery wall and its application to clinical data," in IEEE Eng. in Medicine and Biology Society, 29th Annual International Conference. 2007: Lyon.

[3] Stoitsis, J., S. Golemati, and S. Nikita, "A Modular Software System to Assist Interpretation of Medical Images-Application to Vascular Ultrasound Images." IEEE Trans. on Instrumentation and Measurement, 2006. 55(6): p. 1944-1952.

[4] Bang, J., et al., "A New Method for Analysis of Motion of Carotid Plaques from RF Ultrasound Images." Ultrasound in Medicine \& Biology, 2003. 29(7): p. 967-976.

[5] Meairs, S. and M. Hennerici, "Four-Dimensional Ultrasonographic Characterization of Plaque Surface Motion in Patients with Symptomatic and Asymptomatic Carotid Artery Stenosis." Stroke, 1999(30): p. 1807-1813.

[6] Barron, J.L., D.J. Fleet, and S.S. Beauchemin, "Performance of Optical Flow Techniques." Int. Journal of Computer Vision, 1994. 12(1): p. 43-77.

[7] Horn, B. and B. Schunck, "Determining Optical Flow." Artificial Intelligence, 1981. 17(2): p. 185-204.

[8] Nagel, H.H. and W. Enkelmann, "An investigation of smoothness constraints for the estimation of displacement vector fields from image sequences." IEEE Trans. on PAMI, 1986. 8: p. 565-593.

[9] Negahdaripour, S., "Revised definition of optical flow: integration of radiometric and geometric cues for dynamic scene analysis." IEEE Trans. on Pattern Analysis and Machine Intelligence, 1998. 20(9): p. 961-979.

[10] Pock, T., et al., "A Duality Based Algorithm for TV- L1-Optical-Flow Image Registration. Lecture Notes is Computer Science." Vol. 4792/2007. 2007: Springer Berlin. 511-518.

[11] Ng, L. and V. Solo. "A data-driven method for choosing smoothing parameters in optical flow problems." in International Conference on Image Processing. 1997.

[12] Krajsek, K. and R. Mester. "On the equivalence of variational and statistical differential motion estimation." in IEEE Southwest Symposium on Image Analysis and Interpretation. 2006.

[13] Krajsek, K. and R. Mester. "A Maximum Likelihood Estimator for Choosing the Regularization Parameters in Global Optical Flow Methods." in IEEE International Conference on Image Processing 2006.

[14] Anandan, P., "A Computational Framework and an Algorithm for the Measurement of Visual Motion." Int. Journal of Computer Vision, 1989. 2(3): p. 283-310.

[15] Bruhn, A. and J. Weickert, "Lucas/Kanade Meets Horn/Schunk: Combining Local and Global Optic Flow Methods." Int. Journal of Computer Vision, 2005. 61(3): p. 211-231.

[16] Bruhn, A., J. Weickert, and C. Schnorr, "Combining the Advantages of Local and Global Optic Flow Methods." Lecture notes in computer science, 2002. 2449/2002: p. 454-462.

[17] Gibson, D. and M. Spann, "Robust optical flow estimation based on a sparse motion trajectory set." IEEE Transactions on Image Processing, 2003. 12(4): p. 431-445.

[18] Murillo, S., et al. "Atherosclerotic Plaque Motion Analysis from Ultrasound Videos." in Fortieth Asilomar Conference on Signals, Systems and Computers 2006.

[19] Murillo, S., et al. "Atherosclerotic Plaque Motion Trajectory Analysis from Ultrasound Videos." in IEEE Int. Special Topic Conf. on Inform. Technology in Biomedicine. 2006. Ioannina, Greece.

[20] Murray, V., et al. "An AM-FM model for Motion Estimation in Atherosclerotic Plaque Videos." in Forty-First Asilomar Conference on Signals, Systems and Computers. 2007.

[21] Stoitsis, J., et al., "Carotid Artery Motion Estimation from Sequences of B-mode Ultrasound Images: Effect of Dynamic Range and Persistence," in 2006 IEEE Int. Workshop on Imaging Systems and Techniques. 2006.

[22] Lucas, B.D. and T. Kanade. "An Iterative Image Registration Technique with an Application to Stereo Vision." in International Joint Conf. on Artificial Intelligence. 1981.

[23] Loizou, C.P., et al., "Comparative Evaluation of Despeckle Filtering In Ultrasound Imaging of the Carotid Artery." IEEE Trans. on Ultrasonics, Ferroelectrics, and Frequency Control, 2005. 52(10): p. 1653-1668.

[24] Horn, B., "Robot Vision." The MIT Electrical Engineering and Computer Science. 1986: McGraw-Hill.

[25] Horst, R.a., P.M.a. Pardalos, and N.V. Thoai, "Introduction to Global Optimization." Nonconvex Optimization and Its Applications, ed. P. Pardalos. Vol. 48. 2000: Kluwer Academic. 